\title{
Nihilisme identitaire dans Lord of the Flies de William Golding et $A$ Slight Ache de Harold Pinter
}

\author{
Dr. Maurice Gning \\ Enseignant-chercheur, Université Gaston BERGER de Saint-Louis, Sénégal \\ Doi: 10.19044/esj.2017.v13n32p109 URL:http://dx.doi.org/10.19044/esj.2017.v13n32p109
}

\begin{abstract}
Two works of the mid-twentieth-century British literature form the corpus of this study, namely Lord of the Flies (1954) by the English William Golding and A Slight Ache (1961) by his contemporary and compatriot Harold Pinter. Based on the issue of nihilism as defined by Nietzsche and on the poststructuralist theory of the death of the subject, it aims to analyze how the two postmodern writers, Golding and Pinter, stress the emptiness of the human identity resulting from the collapse of the Western culture.

The analysis shows that, in order to reveal this identity vacuity, the two authors make use of strategies at first sight different, but that prove to be basically similar. This identity emptiness is beforehand expressed by the emptiness of the fiction space, the isolation of characters and the justified absence of traditional points of reference that could constitute the base of the societies they attempt to form. The predictable collapse of these societies discloses the strange face of the individual behind it, and unveils the kingdom of nothingness foregrounded, in both works, by the image of darkness and chaos.
\end{abstract}

Keywords: Emptiness, nihilism, post structuralism, identity, culture, space, Western world

\section{Résumé}

Cette étude porte sur deux œuvres de la littérature britannique de la seconde moitié du $20^{\text {ème }}$ siècle, à savoir Lord of the Flies (1954), un roman de l'Anglais, William Golding, et une pièce théâtrale, A Slight Ache (1961), de son contemporain et compatriote, Harold Pinter. Prenant appui sur le nihilisme de Nietzsche et sur la théorie poststructuraliste de la mort du sujet rationnel, elle cherche à analyser comment Golding et Pinter, deux écrivains postmodernes, mettent en évidence le vide de l'identité humaine qui s'est révélé suite à l'écroulement du fondement culturel de l'Occident.

L'analyse conclut que les deux écrivains usent de stratégies, au premier abord, différentes, mais, au fond, fort comparables pour rendre compte de cette 
vacuité identitaire. Celle-ci est d'abord exprimée par le vide de l'espace fictionnel, l'état de solitude des personnages et l'absence bien justifiée de repères identitaires réels sur lesquels pourraient s'appuyer les sociétés qu'ils tentent de construire. L'effondrement prévisible de ces sociétés révèle la face singulière de l'homme qui en est la cause, et inaugure le royaume du néant mis en évidence, dans les deux œuvres, par l'image de l'obscurité et du chaos.

Mots-clés : Vide, nihilisme, poststructuralisme, identité, culture, espace, Occident

\section{Introduction}

Le dramaturge Harold Pinter et le romancier William Golding, deux lauréats du prix Nobel de littérature, font partie des écrivains les plus marquants de la littérature britannique de la seconde moitié du $20^{\text {ème }}$ siècle. Leurs œuvres portent les marques des graves inquiétudes de l'époque qui les a produites et sont généralement classées dans le courant postmoderne. De façon globale, les deux artistes y posent, avec une sagacité rare, la question de l'identité humaine que les atrocités traumatisantes de la Deuxième Guerre mondiale ${ }^{55}$ ont complètement bouleversée et remise au goût du jour.

Golding estime que le mal qui mine l'existence humaine va au-delà du système qui semble l'engendrer et s'enracine dans la profondeur même de la nature humaine. Cette découverte lui aura fait autant de mal que la guerre, comme 1'explique Foster ;

The war alone was not what appalled him, but what he had learnt of the natural - and originalsinfulness of mankind did. It was the evil seen daily as commonplace and repeated by events it was possible to read in any newspaper which, he asserted, were the matter of Lord of the Flies. The war could be regarded as the catalyst which released an already present evil. People possessed

\footnotetext{
${ }^{55}$ Les deux écrivains ont, de leurs propres aveux, subi le traumatisme de la Deuxième Guerre mondiale. Golding y a activement pris part sous la bannière de la Marine Royale, notamment dans le cadre des opérations de libération de la France occupée par les Nazis. La farouche opposition de Golding à l'idéologie raciste et impérialiste est bien connue. Quant à Pinter, âgé seulement de 9 ans à l'éclatement de la guerre, il faisait partie de ces milliers d'enfants londoniens qu'on évacuait dans les banlieues de l'Angleterre notamment à Cornwall et à Reading, comme justement les jeunes écoliers dans Lord of the Flies de Golding. Lorsque Pinter regagna la maison familiale à Londres, la guerre n'était pas terminée. Les bombardements des raids aériens se poursuivaient. Sa vie et celle des autres étaient en danger, comme il l'expliquera plus tard. Il a également été bouleversé par la brutalité avec laquelle les nazis ont tué certains de ses amis lors de la guerre. Enfin, Juif, Pinter a vécu la violence liée à l'antisémitisme dans le populeux quartier juif de l'East End de Londres où il a grandi.
} 
this trait in a fundamental and permanent fashion it could emerge at any time and under any condition (Foster 10)

A l'image de Golding, Pinter voit dans la nature humaine la source de l'insécurité existentielle. Il considère que l'homme est habité par un désir insatiable de domination qui fait que les relations sociales sont toujours heurtées et la vie quotidienne ponctuée de menaces. Ce que révèlent aussi ces rapports conflictuels qui prennent plusieurs formes, c'est le caractère particulièrement étrange de l'homme que trahissent son langage et ses gestes de tous les jours.

Comme la plupart des écrivains d'après-guerre, Golding et Pinter se font les porte-paroles de toute une génération déçue par les promesses du modernisme et qui entrent tristement dans l'ère du nihilisme dont Nietzsche avait annoncé l'avènement un demi siècle auparavant. Le nihilisme, au sens où l'emploie Nietzsche, renvoie d'abord à cette attitude de dépréciation de l'existence et de l'homme par ce dernier lui-même au profit des «idoles », c'est-à-dire de toutes les valeurs idéologiques de l'Occident depuis l'Antiquité jusqu'aux temps modernes : le Monde Intelligible de Platon dont le nôtre n'est que pâle reflet, le Christianisme avec son Dieu Créateur et Parfait auquel l'homme imparfait doit se soumettre, la morale chrétienne avec son lot d'interdictions et son appel à une prétendue humilité et amour, la science dans sa prétention à être vérité, source de bonheur et qui a fini d"'ailleurs par tuer Dieu en le reléguant au second plan. Le nihilisme désigne ensuite la douloureuse conscience du sentiment du néant, suite au naufrage des « idoles », à l'écroulement des fondements illusoires dont le plus représentatif est Dieu, tristement « mort ». L'homme comme créature divine, être rationnel, autonome, transparent et capable de rendre compte de ses gestes s'est évanoui consécutivement à la mort de son Créateur. Il est finalement mort, nous apprend Foucault. Et l'autopsie que lui et ses pairs poststructuralistes ont faite révèle une réalité renversante. Elle nous apprend, en effet, que cet homme libre ayant prise sur lui et sur le monde n'a jamais existé. Il s'agit plutôt d'un fantôme dont on vient de se rendre compte de la nature. En fait, c'est un être vide sous-tendu par des structures qui déterminent son mode de vie et sa pensée. Arraché à son socle culturel, sociétal et idéologique, l'homme se dévoile comme un néant à l'image de l'existence. C'est ce vide qui se révèle comme le fondement de l'identité des personnages des deux œuvres à l'étude que nous désignons essentiellement sous le concept de «nihilisme identitaire », ce qui n'est pas sans rappeler le nihilisme de Nietzsche. En nous fondant sur ce nihilisme dans sa double dimension susmentionnée, mais aussi sur le principe poststructuraliste de la mort de sujet rationnel, nous nous proposons, dans cette étude, de voir comment Harold Pinter et William 
Golding, respectivement dans A Slight Ache et Lord of the Flies, dévoilent le vrai visage de l'homme quand tombe le masque culturel qui le cachait.

\section{L'espace fictionnel et le vide ontologique}

A Slight Ache met sur scène un couple, Edward et Flora, retiré dans leur appartement à côté duquel se tient un vieillard étrange apparemment vendeur d'allumettes. Le constat de la présence de l'inconnu met fin à la vie paisible du couple, spécialement celle d'Edward qui a du mal à cacher son inquiétude, en dépit des tentatives de sa femme de le calmer. Ayant essayé sans succès de percer le mystère du vieillard visiblement sourd-muet, Edward finit par perdre sa femme au profit du vieil homme. En effet, Flora invite l'homme à vivre définitivement avec elle dans la maison et remet les boîtes d'allumettes qu'il détenait à Edward à qui elle demande de sortir et prendre la place de l'homme.

Dans Lord of the Flies, un avion évacuant un groupe de jeunes écoliers anglais âgés de 6 à 12 ans, dans un contexte de guerre nucléaire, s'est écrasé sur une île déserte, causant la mort du pilote et de tous les adultes accompagnants. Laissés seuls face à eux-mêmes, les enfants tentent d'organiser leur nouvelle société autour de leur chef élu, Ralph, avec ses collaborateurs Piggy et Simon. Cependant, les règles établies sont rapidement violées avant d'être détruites, et avec elle la jeune société, par un groupe de jeunes rebelles sous la direction de Jack qui se plait à perpétrer des actes de barbarie, transformant ainsi l'île vierge en une véritable jungle et les jeunes anglais définitivement en barbares.

Par-delà leurs orientations différentes, les deux œuvres révèlent la coprésence de deux mondes dont l'un est issu de l'autre, l'y ressemble et en même temps s'y oppose. Lord of the Flies met en opposition la société formée par les jeunes dans l'île et la société anglaise qui l'a secrétée et qui est ici incarnée notamment par Ralph, Piggy et Simon. Comparativement, A Slight Ache, comme d'ailleurs la presque totalité des pièces de Pinter, met face-àface deux univers : un monde intérieur fermé, symbolisé par l'espace privé où vivent Edward et Flora, et un monde extérieur ouvert, investi ici par le mystérieux vieillard.

L'espace ouvert dans la pièce de Pinter et la société anglaise qui apparaît en filigrane dans le roman de Golding symbolisent l'ancien monde avec ses valeurs devenues caduques, le monde incertain et violent dans lequel l'homme est réduit à adopter des stratégies de survie face à la menace quotidienne. A cet univers d'insécurité, ce grand monde de danger, s'oppose l'espace de sécurité, le petit monde privé des personnages des œuvres à l'étude. C'est dire que ces petits mondes, tout en matérialisant la faillite des valeurs du monde réel, sont la négation de celui-ci et se posent comme de nouvelles alternatives. De ce fait, ils constituent le symbole du désastre, de la 
coupure ontologique, de la rupture du lien qui jadis unissait l'homme à luimême et à son environnement, autrement dit de la crise identitaire consécutive à l'écroulement de tous les systèmes de valeurs qui fondaient le monde occidental.

L'appartement, dans A Slight Ache, est donc un nouveau monde, une nouvelle existence, tout comme l'est l'île vierge dans Lord of the Flies qui évoque le Jardin d'Eden où la vie aurait commencé, d'après la révélation biblique. Edward et Flora ont le nécessaire pour bien vivre dans leur milieu intime et même un jardin pour s'épanouir ; un jardin dont la verdure, renforcée par la masse de fleurs qui y apparaissent, rappelle l'île aux nombreux arbres fruitiers et cochons sauvages servant de nourriture à Ralph et ses camarades. Que la sécurité et 1'existence des personnages dépendent de 1'espace qu'ils occupent met en évidence le rôle hautement symbolique que joue celui-ci. En effet, l'espace sous-tend et reflète l'identité des personnages. Rien de surprenant dès lors qu'ils revendiquent avec fierté d'en être les propriétaires exclusifs. Edward rappelle, non sans inquiétude, que la maison à côté de laquelle se tient l'homme inconnu est bien la sienne : "It's my own house, isn't it ....It's my own gate" (98). Les jeunes dans Lord of the Flies se réjouissent aussi de constater que l'île, riche en nourriture sauvage, est inhabitée et qu'ils peuvent en faire leur propriété exclusive : "This is our island. It's a good island "(38), affirme le chef du groupe. L'identité des personnages ne saurait donc être mieux appréhendée qu'à partir des traits spécifiques de leur espace d'évolution, étant entendu que celui-ci est le garant de leur existence et de leur essence. Or, dans les deux œuvres, l'espace se révèle particulièrement vide. Ralph nous en donne une idée dans Lord of the Flies lorsque, de retour à une expédition dans l'ile en compagnie de Jack et de Simon, il révèle : "We're on an island. We've been on the mountain-top and seen water all around. We saw no houses, no smoke, no footprints, no boats, no people. We're on an inhabited island with no other people on it" (35). Cette vacuité spatiale dans Lord of the Flies n'apparaît pas de façon nette dans A Slight Ache; elle est plutôt suggérée et incarnée par les nombreux objets familiaux qui envahissent l'espace de jeu, mais qui semblent être vidés de toute portée symbolique, donnant ainsi au décor une impression de vide, comme le rappelle fort à propos Liza Kharoubi:

La scène du théâtre d'Harold Pinter présente souvent l'intérieur meublé d'une maison au premier abord tout à fait ordinaire - lits, tables, et chaises organisent un espace presque étonnant de réalisme, tandis que les portes et les fenêtres suggèrent qu'il existe également un monde extérieur. Tout indique le confort et chasse l'inquiétude claustrophobe d'un public assis dans le noir. Pourtant, cette transparence du décor, sans fioriture esthétique ni anomalie architecturale, donne 
une impression de vide. Il ne s'agit pas vraiment de minimalisme délibéré, mais d'une froideur ambiante qui circule entre les meubles et accuse les distances entre les quatre coins de la scène. (Kharoubi 1)

Le vide de l'espace met les personnages qui s'y meuvent face à euxmêmes. Ils sont littéralement coupés du reste du monde et placés dans un contexte où les valeurs sociales, religieuses, politiques ou idéologiques sont pratiquement absentes. Ils sont dans le vide et se découvrent comme des êtres vides. Ils sont alors à «l'Ere du vide » pour faire allusion au titre d'un ouvrage de Gilles Lipovetsky. L'espace fictionnel dans Lord of the Flies et A Slight Ache, précisément dans son aspect vague, remplit ainsi une fonction allégorique qui est celle de connoter le néant existentiel faisant suite à la dissolution de tous les repères de l'occident. Par analogie, les personnages occupant cette aire neutre représentent le sujet occidental arraché à tous ses fondements culturels et devenu un néant incarné. C'est ce qui explique, en partie, tout le flou des personnages. Ils manquent de profondeur psychologique et ne peuvent être intégrés dans des catégories sociétales bien définies.

Cette absence d'ancrage identitaire est l'un des traits distinctifs des pièces de Pinter. Tout un mystère entoure ses personnages qui ont l'air de sortir du néant et sont très réticents à dévoiler leur vie et le fond de leurs pensées. A titre d'exemple, nous ne connaissons rien du passé de Flora et d'Edward; nous ne savons pas s'ils sont mariés ou pas. Comme Edward et certainement Flora, nous ignorons tout du vieillard vendeur d'allumettes qui se présente devant leur maison. Le spectateur ne sait pas grand chose des personnages de Pinter, en dehors des rares révélations qu'ils font de leur vie présente et antérieure; des révélations d'ailleurs fort douteuses, dans la mesure où ils se servent des mots moins pour dévoiler que pour cacher ou taire leurs sentiments et vécus.

Il en va de même des jeunes garçons dans Lord of the Flies qui sont littéralement descendus du ciel. Leur passé individuel est inconnu. Leurs noms le sont aussi au début, comme l'atteste la récurrence du terme anonyme 'the boy' que le narrateur utilise pour les présenter tour à tour. Le roman s'ouvre d'ailleurs par une description vague des personnages : " the boy with fair hair lowered himself down the last few feet of rock an began to pic his way" (7). "Thefat boy looked startled" (8). Ce n'est qu'un peu plus tard que le lecteur apprend que "the boy with fair boy" s'appelle Ralph, et "the fat boy", Piggy. Ralph et Piggy, errant dans l'île aux aspects paradisiaques, sont comparables à Adam et Eve au Jardin D'Eden, d'autant plus que ce sont eux qui, à l'image du couple originel, vont donner naissance à la société des petits garçons en travaillant à les réunir.

Cette image de l'homme renvoyé à ses origines bibliques traduit une envie quasi obsessionnelle chez Golding de faire la généalogie du mal, de 
retrouver la racine du péché qui a corrompu l'homme en le replaçant dans le paradis, ce beau cadre avec lequel l'être humain, vide de tout péché, était en parfaite harmonie. Malheureusement, c'est au fond de cette innocence originelle que s'est enfoui le mal qui va surgir et tout ravager. L'homme est donc venu au monde avec le mal. Il faut peut être en vouloir à son créateur. Mais de sa chaise roulante où il est définitivement cloué, Hamm, le personnage principal de Endgame de Beckett, constate de façon blasphématoire que Dieu n'existe pas : “The bastard! He doesn't exist!" (Beckett 38). Il faut donc s'en prendre aux procréateurs, comme le fait Hamm qui traite son père de fornicateur et à qui il ne peut pas pardonner de l'avoir engendré. Comparativement, le barbarisme des enfants qui clôt Lord of the Flies ne peut pas être fondamentalement imputable à l'enfance, à l'absence de supervision des adultes. Ce que semblent oublier Piggy et Ralph qui font constamment référence au monde des adultes et qui estiment que le salut du groupe n'est possible que lorsqu'il y retourne, c'est que ce monde est loin d'être une référence. Comme le leur, il est déchiré par la violence et la barbarie, ce qui d'ailleurs leur a valu d'être évacués et d'atterrir accidentellement dans l'île déserte, qu'ils ont transformée en une seconde Angleterre en proie à une guerre nucléaire. C'est peut-être pour nous convaincre que le mal n'est pas lié à une question d'âge, mais essentiellement au genre humain que Golding choisit de représenter l'humanité dans son deuxième roman Inheritors (1955), mais toujours comme génératrice du mal. Dans ce livre, l'auteur montre comment l'homo sapiens, la première espèce humaine dotée d'intelligence, donc ancêtre direct de l'homme moderne, a détruit la vie harmonieuse des dernières tribus de l'homme de Neandertal.

Golding fait recours aux mythes des origines de l'homme pour mettre en lumière la nature primitive de celui-ci, le vide et le mal naturel qui le caractérisent. Pinter adopte une stratégie bien différente. Il retrouve ce vide dans la vie présente, dans la réalité quotidienne de son Angleterre natale. En effet, comme s'il partageait l'idée de Blaise Pascal ou de Schopenhauer selon laquelle c'est dans la solitude que se révèle la vacuité humaine, Pinter essaie de saisir ce vide ontologique à un moment particulier de la vie de l'individu, c'est-à-dire quand, dans l'intimité de sa chambre ou de sa maison, il est débarrassé de tous les artifices et soucis sociaux, libéré de toutes les contingences matérielles et des préoccupations idéologiques et faisant face à lui-même en tant que présence contingente dans le monde, ce fameux Dasein Heideggérien. C'est pourquoi, s'il est question chez Golding d'île déserte, de l'homme de Neandertal, de l'homo sapiens ou de l'enfant qui n'a pas encore complètement intériorisé les valeurs de la société, chez Pinter nous sommes face à l'espace bien connu de la chambre ou de la maison, à l'homme qu'on rencontre dans n'importe quelle rue de Londres et peut-être même partout dans le monde. C'est bien l'homme adulte et reconnaissable, mais saisi dans un état 
particulier qui ne le diffère pas trop de l'enfant. Au vrai, le vide culturel de l'enfant encore innocent dans le roman de Golding est fort comparable à celui de l'adulte culturellement dénudé qu'on retrouve dans la pièce de Pinter. Le sujet dans les deux œuvres est ainsi « néantisé», pour reprendre un terme de Lacan. Il n'est qu'une «enveloppe creuse», comme dirait Baudrillard. Toutefois, l'homme semble avoir horreur du vide, surtout quand ce rien se trouve être le socle même de sa nature et existence. Il faut donc agir pour masquer ce nihil, comme semble le suggérer Piggy qui répète ceci: "We got to do something" (15).

\section{Le masque social face au vide de la nature humaine}

Etant donné que c'est dans la solitude que s'exprime ce vide dans sa plénitude, c'est alors contre cette situation existentielle qu'il sied de se prémunir. Il s'agit d'activer le levier social, de chercher à s'unir avec son semblable, d'inventer des valeurs communes qui garantiraient le bon vouloir vivre ensemble, bref de créer une société et donc une identité. C'est à cela que s'appliquent exactement les deux premiers garçons apparus dans Lord of the Flies, à savoir Ralph et Piggy. "We got to find the others" (15), persiste Piggy. Ils entreprennent de rassembler tous les garçons égarés dans l'île, d'identifier chacun par son nom, d'élire un chef et d'établir des règles de vie sous la surveillance de Ralph, le chef élu. Il est question pour les deux jeunes de tenter de reproduire instinctivement les schémas sociaux de leur nation d'origine dont paradoxalement les adultes essaient de les mettre à l'abri, car ayant atteint toutes ses limites. L'impérieuse nécessité de se libérer de la conscience douloureuse de son propre vide semble également commander la relation entre Flora et Edward. Bien qu'ils soient unis, du moins en apparence, par un lien conjugal, tout les sépare, réduisant ainsi chacun à un état d'isolement irrémédiable.

Autant le vide est effroyable, autant il est inévitable. Les personnages sont dans l'incapacité absolue de s'en libérer pour la simple raison qu'on ne peut pas échapper à soi-même. Loin de leur permettre de se défaire du néant, les actes qu'ils posent les y enfoncent davantage. Les deux œuvres s'achèvent sur une note de chaos total, attestant ainsi l'inefficacité et l'échec de l'entreprise identitaire dans laquelle s'engagent vainement les protagonistes. Rose et Flora sont définitivement séparés. La société que cherchaient à former Ralph et Piggy s'est entièrement effondrée. La violence et la barbarie ont pris le dessus sur les normes sociales. Les garçons sont revenus à la case de départ, au désordre initial se faisant écho de la confusion qui prévaut dans leur Angleterre natale.

En fin de compte, et c'est la conclusion pessimiste à laquelle semble arriver Golding, toutes les valeurs établies par l'homme sont systématiquement détruites par ce dernier. Tiger nous en donne la raison : "So 
the boys try to construct a civilization on the island; but it breaks down in blood and terror because the boys are suffering from the terrible dis- ease of being human"(Tiger 85). La maladie d'être humain que Tiger évoque est telle que Levi-Strauss considère l'homme comme une machine de destruction travaillant efficacement à l'effondrement de sa propre civilisation. Dans Tristes Tropiques, il affirme que les sociétés humaines sont vouées à l'anéantissement. S'il en est ainsi, c'est parce que la société -comme un ensemble de normes et de valeurs structurant et rendant possible la vie en communauté - serait aux antipodes de la nature humaine que Freud, Spinoza, Schopenhauer et Nietzsche ont généralement décrite comme désir. De ce point de vue, la société est par essence nihiliste au sens nietzschéen du terme. Elle nie la nature de l'homme en se posant comme une entrave à l'expression du désir de l'individu, ce ça freudien, ce « vouloir-vivre » schopenhauerien ou encore cette «volonté de puissance» nietzschéenne, bref cette pulsion irrationnelle qui définit l'individu. Autant dire que la vie communautaire, gage d'identité sociale (adhésion et identification à des valeurs communes d'un groupe social), repose sur un fondement fragile puisqu'elle n'est qu'une négation de l'identité ontologique, de la nature humaine (un être vide, un être de désir irrationnel), la vraie identité, est-on tenté de dire; une identité ontologique de nature destructrice à l'expression de laquelle rien ne semble pouvoir désormais s'opposer, d'autant que les structures chargées de la dompter se sont effondrées les unes après les autres. Cela est particulièrement valable pour les jeunes déchaînés dans Lord of the Flies qui, sous le joug des pulsions de mort freudiennes, sont engagés dans une entreprise de destruction de toutes les valeurs qui humanisent l'individu. En revanche, chez Pinter, il s'agit moins d'engager une bataille consciente contre les valeurs sociales que d'une difficulté à maintenir le contact avec son partenaire au sein de l'espace privé, compte tenu de la vacuité des mots et la menace d'intrusion de l'inconnu. Mais dans l'un ou dans l'autre cas, la société s'avère être une construction sans fondation solide.

\section{Les mots et la coquille : une société bâtie sur le néant}

Pinter et Golding mettent tous les deux à nu ce fondement fragile de la société qui assure un semblant d'identité culturelle à leurs personnages. La relation entre Edward et Flora au sein de leur espace privé ainsi que la société que tentent de former Ralph et ses collaborateurs sont bâties sur le néant. Dans la pièce de Pinter, ce néant est exprimé par les mots vides de sens qui constituent le seul moyen pour Flora et Edward de maintenir leur relation. Aussi se livrent-ils à un dialogue insignifiant, insipide et plat. L'échange suivant qui ouvre la pièce l'exemplifie:

Flora: Have you noticed the honeysuckle this morning? 
Edward : The what?

Flora : The honeysuckle.

Edward : Honeysuckle? Where?

Flora :The back gate, Edward.

Edward : Is that honeysuckle? I thought it was ...

convolvulus, or something.

Flora : But you know it's honeysuckle.

Edward : I tell you I thought it was convolvulus.

[Pause.] (94)

Ce genre de dialogue qui traverse l'œuvre théâtrale de Pinter, en particulier, et en général celles des dramaturges dits de l'absurde, tels que Beckett, Ionesco, Genet et Adamov, témoigne de ce qu'il est convenu d'appeler la crise du langage. Cette crise s'annonce dans les réflexions de Saussure qui démontrent que le signe linguistique est arbitraire, conventionnel, autrement dit, qu'il n'y a pas de lien naturel entre le mot et son référent, entre le signifiant et le signifié. Nietzsche corrobore ce point de vue. Il va même plus loin en soutenant que le langage, parce qu'il ne produit que des représentations métaphoriques de tout ce qui existe, ne saurait accéder à la vérité, c'est-à-dire l'expression adéquate de cette réalité :

Comparées entre elles, les différentes langues montrent qu'on ne parvient jamais par les mots à la vérité, ni à l'expression adéquate : sans cela il n'y aurait pas de si nombreuses langues. La «chose en soi » (ce serait justement la pure vérité sans conséquence), même pour celui qui façonne la langue, est complètement insaisissable et ne vaut pas les efforts qu'elle exigerait. Il désigne seulement les relations des choses aux hommes et s'aide pour leur expression des métaphores lesplus hardies (...). Nous croyons savoir quelque chose des choses elles-mêmes quand nous parlons d'arbres, de couleurs, de neige et de fleurs et nous ne possédons cependant rien que des métaphores des choses, qui ne correspondent pas du tout aux entités originelles.(Nietzsche 179)

A cette remarque fracassante de Nietzsche, s'ajoute le coup dur que le psychanalyste Freud et les poststructuralistes ont porté au langage en dévoilant que celui-ci n'est que l'expression d'un ensemble de structures qui gouvernent la conscience humaine. Pour Foucault par exemple, le langage est un véhicule de pouvoir. Ce n'est donc pas l'homme qui parle, mais ce sont des structures mentales qui parlent en lui. Déjà en 1871, dans sa correspondance à Georges Izambard, Arthur Rimbaud écrivait ceci : «c'est faut de dire je pense : on devrait dire on me pense ». Et Lacan de préciser plus tard que « ça parle », une 
formule devenue célèbre. Le pouvoir de dire « je » dont Kant disait qu’il élève l'homme au-dessus de tous les autres vivants lui est catégoriquement nié par ces penseurs. Après l'individu, cet être coupé de toutes ses racines pour ne devenir qu'un vide en marche, c'est au tour du langage d'être vidé de sa substance, de sa capacité à dire le réel, à interpréter le monde. A quoi peut-il servir dès lors sinon à remplir le vide ? C'est en tout cas la seule fonction que lui assignent les sujets errants qui peuplent les pièces de Beckett. C'est aussi, en partie ${ }^{56}$, le sens qu'il prend dans le théâtre de Pinter. A titre d'exemple, la discussion entre Edward et Flora, qui précède le constat de la présence de l'inconnu, est dépourvue de toute fonction référentielle. Elle ne renvoie à rien de particulier. Elle est plutôt réduite à sa simple dimension phatique, c'est-àdire servant à maintenir et prolonger le contact entre eux, sans le moindre souci de livrer une quelconque vraie information; d'où son extrême insignifiance qui ne fait que refléter le vide ontologique de ceux qui s'en servent.

Outre la banalité dont il est porteur, le langage est le champ d'expression de toutes les ambiguïtés. Il ne peut d'ailleurs en être autrement. En raison de son incapacité à traduire convenablement le réel, il devient, comme le dit Pruner, " le point extrême de la subjectivité » (Pruner 128). L'interprétation des mots est ouverte à tous vents et chaque être demeure figé dans sa propre compréhension. Il en résulte, comme nous le constatons fréquemment dans les pièces de Pinter, désaccord et incompréhension qui donnent lieu à des querelles parfois violentes. Meg, dans the Birthday Party, n'apprécie pas le terme "succulent" (1983:7) que son interlocuteur Stanley utilise. Dans the Dumb Waiter, Ben ne tolère pas que son ami Gus dise "put on the kettle" (1983:141) et le menace avec un couteau. Pour lui, 1'expression adéquate est : "light the kettle ". Il y a une différence entre "to sit down" et "to perch "1983 :116) souligne, avec autorité, Mr Sand à l'intention de Mrs Sand dans The Room. Pour lui, il y a «s'asseoir » et « mal s'asseoir ». Et le verbe approprié pour exprimer le fait d'être mal assis est "to perch". Edward fait remarquer à Flora qu'on doit dire "wasps sting" et non "wasps bite "(96) et menace d'abandonner cette dernière si elle persiste dans son «erreur» lexicale:"If you don't stop saying that word I shall leave this table "(96). Source d'incompréhension, car très subjectif, le langage devient ce par quoi les êtres humains prennent conscience de leurs différences, alors qu'il était destiné à les unir. Il rend les personnages davantage sensibles à leur état de solitude, donc au sentiment du nihil. Le caractère faillible du langage ajouté à

\footnotetext{
${ }^{56}$ Le langage dans le théâtre de Pinter est aussi un outil efficace au service de la lutte pour la domination. Comme l'affirme Pinter, il est dangereux de révéler à autrui, surtout à l'inconnu, notre pauvreté intérieure car il peut l'exploiter à tout moment à des fins de domination. Parler devient donc une façon de se taire. C'est à travers le jeu de langage que se révèle d'abord très souvent le face-à-face toujours conflictuel avec l'inconnu dans les pièces de Pinter. C'est le langage du non-dit, de l'esquive.
} 
son aspect vide témoignent de la précarité de l'ordre établi dans l'espace privé et constituent le signe avant-coureur de sa fin.

Si le néant sur lequel s'est édifiée la petite société dans A Slight Ache se donne à lire à travers les mots sans substance, semblables à une coquille, et qui lient Edward et Flora, il est exprimé de façon symbolique dans Lord of the Flies où il prend justement la forme d'une coquille au sens littéral du terme. En effet, c'est à l'aide de cet objet retrouvé par hasard dans l'île que la nouvelle société est formée. Sur conseil du très avisé Piggy, Ralph souffle dans la conque pour attirer tous les garçons et tenir une réunion grâce au bruit fort qu'elle émet : "We can use this to call the others. Have a meeting. They will come when they hear us" (17). La coquille n'est qu'une enveloppe ayant perdu d'ailleurs son rôle de protecteur car séparée du mollusque dont il devait envelopper le corps. Par conséquent, elle ne signifie plus rien et est, en ce sens, l'expression par excellence du néant. Elément fondateur de la société des jeunes, elle n'en est pas moins la régulatrice principale, étant donné que c'est elle qui permet à Ralph de convoquer les réunions (tous les jeunes se retrouvent ensemble dès qu'ils entendent le son de la conque) et règlemente la prise de parole lors des assemblées (Il faut lever la main pour prendre la parole ; seul celui qui a la conque est autorisé à parler et dès qu'il termine, celui à qui elle est remise prend le relais).

\section{L'effondrement de l'humain et l'avènement de l'être obscur}

Tout en s'évertuant à instituer des codes de conduite par le biais du coquillage, les jeunes rescapés laissent apparaître des attitudes qui sont nettement en contradiction avec l'obligation de discipline que requiert toute vie en communauté. Cette image contradictoire de l'individu partagé entre la nécessité de se conformer aux normes établies et le désir de les violer est une illustration du conflit interne freudien entre le 'surmoi' (la société avec ses valeurs normatives) et le 'ça' (le moi en tant que siège des pulsions). Il est évident que l'affaiblissement ou l'annihilation des forces du 'surmoi' entraîne inéluctablement l'expression sans limite des désirs irrationnels du 'ça'. Les garçons de Golding sont dans cette situation où les valeurs sociales n'ont encore aucun poids véritable sur eux, car ne tenant que sur un simple coquillage. Ainsi, on comprend pourquoi ils sont enclins à laisser s'exprimer les pulsions irrationnelles du 'ça'. Or, Freud lui-même nous apprend que cette instance psychique est l'essence même de l'individu. « Là où était le 'ça', ditil, le 'je' doit advenir». Les garçons sont alors inconsciemment engagés dans un processus d'affirmation de leur être véritable, de leur nature, de leur identité ontologique. Cette expression de soi implique nécessairement de se défaire de toute pesanteur sociale, de tout ce qui est acquis, même partiellement, pour être soi-même, c'est-à-dire cet « homme de la nature» ou l'homme à l'état de nature par opposition à « l'homme de l'homme» qui est façonné par la société, 
pour emprunter à Rousseau ses termes. C'est cette exigence d'être soi qui sous-tend le fait que presque tous les enfants se débarrassent de leurs habits pour être nus, au propre comme au figuré. C'est déjà assez significatif que ce soit Ralph, le futur chef, le premier à se dévêtir, avant même que ne soient édictés les principes devant régir la jeune communauté:

He became conscious of the weight of his clothes, kicked his shoes off fiercely and ...pulled off his shirt, and stood there among the skull-like coco-nuts with green shadows...he undid the snake-clasp of his belt, lugged off his shorts and pants, and stood there naked, looking at the dazzling beach and the water. (10)

Le premier enfant apparu grâce au son de la conque se signale par des habits déchirés et on le voit se défaire de son pantalon: "He was a boy of perhaps 6 years, study and fair, his clothes torn... His trousers fell about his ankles; he stepped out of them" (18). De même, les autres enfants arrivés plus tard au lieu de la rencontre sont quasiment nus: "some were naked and carrying their clothes... other half-naked, or more-or less dressed, in school uniforms" (19). Comme la langue, les habits sont la marque d'une identité culturelle. Ils rappellent aux jeunes leur appartenance sociale à l'Angleterre. Toutefois, cette appartenance est remise en cause par le fait que leur initiation culturelle, comme le laisse supposer les uniformes d'écoliers que certains portent encore avec peu de soin, a été brusquement interrompue et qu'ils vivent désormais dans un milieu tout différent auquel ils s'adaptent instinctivement. Les vêtements en loques des enfants sont aussi évocateurs de la culture anglaise mise en lambeaux par une guerre meurtrière et traduisent la nécessité de se libérer de cet héritage culturel obsolète et lourd à porter. Nous comprenons ainsi le désagrément qu'ils causent à Ralph et à ses camarades et leur promptitude à s'en débarrasser et rester nus.

Il y a un parallèle entre la nudité, qui est un motif majeur du roman, et l'île inhabitée dans laquelle se délectent les jeunes garçons. L'île déserte rappelle le vide avant l'avènement de l'homme acteur de changement, avant la civilisation. La nudité renvoie à la naissance, au corps frêle du bébé, donc aux premières heures de la vie humaine avant la socialisation. Nous sommes ici au commencement d'un monde, d'une toute nouvelle existence humaine, comme semble vouloir dire le narrateur: "signs of life were now on the beach " (19). Mis à part Ralph et ses compagnons dont l'effectif se réduit drastiquement, les garçons acceptent cette renaissance en se libérant de leurs vêtements culturels pour être dépouillés à l'image de l'île. Aussi laissent-ils apparaître un visage sans identité sociale. Ils délaissent les réservoirs d'eau de fortune faits avec des coques de coco et, comme des animaux, boivent maintenant directement au fleuve. L'endroit aménagé dans les roches pour se 
soulager est déserté et ils font leurs besoins n'importe où y compris même au sein des cabanes qu'ils ont construites pour y dormir, encore que beaucoup passent la nuit à la belle étoile et ne prennent plus part à la construction de ces abris. La coquille, l'instance normative de leur vie sociale, est brisée. Et Jack, anarchiste irréductible, peut s'écrier triomphalement: "we don't need the conch anymore. We know who ought to say things" (111). Ils ont laissé s'éteindre le feu de signalisation allumé sur la montagne pour attirer un éventuel navire qui pourrait les sauver. Tous les éléments culturels, notamment la conque, les abris, le feu, ainsi que les personnages incarnant le mieux cette culture, à savoir Ralph, Piggy et Simon, sont d'abord affaiblis puis anéantis. Piggy et Simon sont sauvagement tués par le groupe violent que dirige Jack. Ralph est pourchassé et traqué comme un cochon sauvage par ce même groupe avant d'être sauvé par un officier de marine britannique. Piggy avait bien raison de s'interroger auparavant sur leur identité : "What are we ? Humans ? Or animals ? Or savages ?" (99). En tout état de cause, c'est désormais par les termes " animals", "savages" que le narrateur désigne les jeunes déchainés, aux visages peints, qui détruisent tout sur leur passage.

Le processus de naturalisation ou de déculturation semble être achevé. Le culturel meurt pour que vive le naturel. Les valeurs sociales disparaissent pour que réapparaisse le vide qu'elles masquaient, le chaos originel dont parle Foucault, que l'homme, selon lui, a toujours cherché à contrôler. Le familier fait place à l'étrange. La beauté naturelle de l'île est à jamais entachée par le visage affreux de l'homme naturalisé qui y rôde, comme ce terrible monstre qui sort nuitamment pour dévorer les hommes du roi Hrothgar, avant d'être tué par le brave Beowulf dans le plus célèbre poème Anglo-saxon intitulé « Beowulf ». L'humain est anéanti pour que surgisse le "ghost » (98), c'està-dire le fantôme, le méconnaissable, car n'ayant aucune forme distincte. Le feu de la civilisation, tel que le craignaient Ralph, Piggy et Simon, s'est définitivement éteint. "The fire », disait Ralph, " is the most important thing on the island. How can we ever be rescued except by lack, if we don't keep a fire going... we've got to make smoke up there - or die'"(88) (c'est nous qui soulignons). La mort des enfants qu'appréhendait Ralph et qui est malheureusement effective est moins biologique que symbolique. Elle renvoie à l'impossibilité de rentrer à la maison mère au double sens littéral et symbolique. Au plan littéral, rentrer à la maison signifie être ramené au monde des adultes en Angleterre où, ils pensent naïvement, tout est encore sous contrôle. Dans sa dimension symbolique, il implique de maintenir le cordon ombilical avec leur société d'origine en perpétuant ses valeurs au sein du nouvel espace. Toutes ces deux options étant compromises, c'est la mort comme anéantissement de tout espoir de renouer avec l'humain qui advient. C'est la mort du sujet, de «l'homme de l'homme », l'homme responsable moulé par la société. 
Des cendres de l'homme soumis à l'ordre sociétal, renaît l'homme opaque, obscur et imprévisible qui, à lui seul, résume tout ce que la vie a de plus incompréhensible et peut être de plus vil. C'est là aussi où réside le sens des visages peints et méconnaissables de Jack et son gang. Ils ont fait leur cette célèbre injonction de Nietzsche : « deviens ce que tu es ». Le retour triomphal de l'homme avant la civilisation, l'homme primitif sans identité précise qui ne nie pas son vide et le désordre de l'existence, est salué par la chanson victorieuse de Jack et son groupe dont le contenu est aussi effrayant que nihiliste : "kill the pig. Cut her throat. Spill her blood"(74). Il est ensuite figuré par les tenues noires que portent les rebelles en remplacement de leurs uniformes d'écolier et autres habits hérités de leur ancienne civilisation : “... a party of boys, marching approximately in steps parallel lines and dressed in strangely eccentric clothing" (19) ... "each boy wore a square black cap...their bodies, from throat to ankle, were hidden by black cloaks" (21). Enfin, cet homme-néant est aussi et surtout symbolisé par les ténèbres qui envahissent l'île à mesure que les enfants font preuve de barbarie. En effet, dans la symbolique occidentale, les ténèbres (la couleur noire et ses dérivées), qui empêchent de voir, de distinguer et de comprendre, représente l'inconnu, l'étrange et le vide. Elles sont ici associées aux enfants. C'est ce que traduit le fait que l'obscurité les suit partout où ils vont: "The darkness seemed to follow round them like a tide"(132). L'homme est l'incarnation même de l'obscurité. Celle-ci est déjà perceptible chez l'enfant, à l'aube de la vie. Tel est le sens de cette oxymore :" in the drakness of early morning " (105). Parce qu'elle implique l'incertitude, l'obscurité est porteuse de menace. Le narrateur n'en dit pas autrement lorsqu'il spécifie:"Soon the darkness was full of claws, full of the awful unknown and menace " (108). Les plus petits du groupe sont ainsi effrayés la nuit. Ils font des cauchemars, voient le danger partout. Comme les personnages de Pinter, ils considèrent le monde hors de leurs abris comme dangereux : "the world outside the shelter was impossibly dangerous" (108). En réalité, et c'est heureux que Simon l'ait compris et souligné, le danger et la menace résident non pas dans les choses ou un quelconque monstre sur l'île, mais dans l'homme lui-même. C'est l'homme en tant que personnification de l'obscurité, de l'inconnu, de l'incertitude et de l'anarchie qui constitue une menace permanente pour son prochain.

Si les garçons dans Lord of the Flies, à l'exception de Simon, ignorent la réalité que la racine du mal se trouve dans l'homme, les êtres dramatiques de Pinter en sont bien conscients. Tel que présenté ici, l'individu est de nature tellement imprévisible et impénétrable qu'il devient systématiquement un danger pour l'autre. C'est pourquoi, dans l'intimité de leurs maisons, les personnages vivent continuellement dans la peur de l'inconnu qui, craignentils, peuvent à tout moment envahir leur espace de vie et porter atteinte à leur quiétude. Voilà ainsi justifiée la panique d'Edward lorsqu'il se rend compte 
de la présence du vieillard inconnu non loin de la porte de son jardin : "Blast and damn it, he's there, he's there at the back gate" (97), "why ? What is he doing there"? (97) "What in God's name is he doing with a tray full of matches at half past nine in the morning "? (98). Par-delà l'affolement qu'elles expriment, les questions d'Edward, qui seront à jamais sans réponses, soulignent le caractère mystérieux du vieillard et par extension de l'individu.

Les tentatives d'Edward, puis celles de sa femme, de démystifier l'inconnu ne portent aucun fruit. L'homme s'est emmuré dans un silence total. Il est visiblement sourd-muet et aveugle. Aucune identité sociale ne peut lui être collée. Il représente l'homme à l'état brut, sorti de nulle part et marqué du sceau du néant. En ce sens, il peut être considéré comme une autre version de l'homme obscur qui est à l'œuvre dans le roman de Golding, c'est-à-dire l'individu coupé de ses racines métaphysiques et culturelles pour, compte tenu de sa non transparence, de son inaccessibilité, former un avec le vide de l'existence.

A la différence de «l'être-néant » qui se déploie dans le roman de Golding, dont les actes barbares et incompréhensibles n'ont apparemment aucune autre motivation que celle d'assouvir le désir d'être soi, celui qu'on retrouve dans l'œuvre de Pinter semble poursuivre un but. Il est bien connu que l'espace est l'un des principaux enjeux du conflit qui oppose les personnages de Pinter. En effet, dans un monde où « tout est incertain et relatif, où il n'y a pas de point fixe ... où nous sommes entourés par l'inconnu» (Salem $90)$, pour reprendre les mots de Pinter, avoir un espace privé où se cacher devient une nécessité. La chambre ou la maison en tant qu'espace de sécurité devient dès lors source de conflit entre les occupants qui tiennent à leur sécurité, et l'intrus qui est aussi à la recherche d'une place fixe dans le monde mouvant et rempli d'incertitudes. Nous comprenons ainsi davantage l'inquiétude croissante d'Edward: "It used to give me great pleasure, such pleasure, to stroll along through the long grass, out through the back gate, pass into the lane. That pleasure is now denied me. It's my own house, isn't it? It's my own gate" (98).

Plus qu'un simple lieu de sécurité, la chambre ou la maison est essentiellement un espace d'identité. Elle permet à ceux qui l'habitent de nourrir un sentiment de certitude, de constance, d'appartenance à un petit monde connu; une assurance que la vie dans le vide de l'univers ne permet pas d'avoir. C'est ainsi qu'Esslin interprète le sens de l'espace clos dans l'œuvre de Pinter : "The room becomes an image of the small area of light and warmth that our consciousness, the fact that we exist, opens up in the vast ocean of nothingness..." (Esslin 1991 : 235-236). La lutte pour 1'espace qui oppose Edward et le vieil aveugle est alors une lutte pour l'identité. Edward ne souhaite pas être tiré de son «espace-identité » du petit monde certain de sa maison hors duquel il meurt, il se noie dans « l'océan du néant », alors que 
l'inconnu cherche à sortir de son « espace-néant », de l'anonymat des ténèbres du dehors. Il veut retrouver la vue, la lumière et la vie, c'est-à-dire l'identité que seul l'espace familial peut lui garantir. Cette image cristallise la lutte pour la reconnaissance hégélienne entre deux consciences qui cherchent à s'affirmer. Pour Hegel, cette confrontation finit toujours par la mort d'un sujet et la reconnaissance mutuelle des adversaires, l'un comme maitre et l'autre esclave. De ce point de vue, Edward peut être déjà considéré comme le vaincu. Il perd progressivement la bataille. Sa panique, la petite douleur qu'il ressent aux yeux, présage d'un aveuglement imminent, le constat que la distance qui le sépare du vieux - qu'il appelle "My oldest acquaintance. My nearest and dearest. My kith and kin"(110) - s'amenuise, sont autant de signes annonciateurs de sa mort, de la perte de son identité et parallèlement de la renaissance du vieil homme qui, à ses yeux, est en train de changer, de devenir plus jeune : "Even now you look different. Very different" (110)... «You look younger. You look extraordinarily ... youthful " (111), fait-il remarquer à l'homme. C'est avec ce vieux rajeuni, donc né à nouveau, que Flora décide de vivre dans l'appartement après l'avoir baptisé Barnabas et envoyé son désormais ex-mari se balader dans les ténèbres du monde extérieur dans lesquelles se fond systématiquement toute identité:

She enters. Ah, Barnabas. Everything is ready.

[Pause.] I want to show you my garden, your garden. You must see my japonica, my convolvulus ... my honeysuckle, my clematis. [Pause.]

The summer is coming. I've put up your canopy for you. You can lunch in the garden, by the pool. I've polished the whole house for you.

[Pause.] Take my hand. Pause. The MATCHSELLER goes over to her. Yes. Oh, wait a moment. [Pause.] Edward. Here is your tray. She crosses to EDWARD with the tray of matches, and puts it in his hands. Then she and the MATCHSELLER start to go out as the curtain falls slowly.( P111)

Le geste inattendu de Flora démontre encore qu'il est impossible de pénétrer le mystère humain. L'individu est d'abord imperméable à lui-même avant de l'être aux autres, y compris ceux avec qui il vit sous le même toit. D'où l'inutilité de la question "who are you ?"(111) que pose désespérément Edward au vieux.

L'identité dans A Slight Ache, faudrait-il le rappeler, ne se définit pas par rapport à des valeurs, à des croyances, à des principes, à un comportement ou à une provenance, mais en relation à un espace intime. Comme c'est 
souvent le cas dans l'écriture postmoderne qui, avec une pointe d'ironie, dévoile la faillite des métarécits, le «je pense, je suis » de Descartes est ici parodié et devient « j’ai un espace, je suis ». La pensée qui, jusqu’à une époque encore récente, était considérée comme le trait distinctif de l'homme, le fondement de son identité, se désagrège sous le poids du diagnostic alarmant des poststructuralistes qui révèlent le fond inconscient la conditionnant.

Que la maison qui n'est qu'un bien matériel, donc très précaire, tienne lieu d'identité ne fait que mettre en évidence le caractère fragile des nouveaux référentiels identitaires qui caractérisent l'individu postmoderne. L'identité ou l'identification de cet individu est fonction de références très mobiles. Pour le sociologue Jean-Claude Kaufmann, « l'identification ne serait même que la recherche permanente du sens de sa propre vie par un individu qui trouverait dans cette quête la possibilité de se détacher de sa socialisation présente pour s'évader vers des réalités imaginaires et fugaces »(Guy 1). On assiste ainsi à la naissance de celui qu' on pourrait appeler « l'homme-papier », autrement dit un être sans fondement identitaire solide qui change au gré des circonstances. Pour Foucault, l'individu postmoderne est un moi impuissant perpétuellement soumis à des forces externes changeantes, comme le rappelle El Diwany : "For Foucault, the postmodern man is no more the transcendental subject that can induce a change depending on the power of his intellect, but rather a powerless 'self' that is constantly open to transformation by other forces" (El Diwany 86). La nature imprévisible des personnages de Pinter, qui surprennent toujours par leurs comportements très variables, pourrait donc être expliquée par leur soumission à des forces externes capricieuses. Dans cette perspective, elle témoigne de l'extinction du sujet moderne dans sa prétention à être autonome et transparent.

\section{Conclusion}

William Golding et Harold Pinter projettent, dans leurs œuvres littéraires, les obsessions de l'homme contemporain, les graves menaces qui pèsent sur le genre humain après la Shoa, Hiroshima et Nagasaki. Ils semblent partager l'idée que le mal qui ronge l'existence est plus lié à la nature de l'homme qu'à un quelconque système. C'est donc cette nature cachée de l'homme qu'ils cherchent inlassablement à mettre en exergue dans leurs différentes œuvres littéraires.

Cependant, comme on peut le constater dans Lord of the Flies et $A$ Slight Ache, les approches et choix esthétiques des deux auteurs sont à la fois différents et similaires. Pour retrouver cette nature humaine, Pinter ne fait recours ni au mythe ni à des considérations irréalistes. Bien au contraire, il va s'inspirer d'un quotidien très simple : deux personnes dans leur espace de vie privé. Golding, quant à lui, imagine une scène relativement mythique : des enfants qui ont survécu à un crash d'avion dans un contexte de guerre et qui 
se retrouvent sur une île inhabitée où ils doivent se débrouiller sans l'aide des adultes.

En plaçant leurs personnages dans des espaces où ils n'ont aucun contact avec le reste du monde, où aucun poids social ou idéologique ne pèse sur eux, Golding et Pinter les dépouillent culturellement et dévoilent tout le vide qui sous-tend leur identité. Chez les deux auteurs, ce vide est d'abord symbolisé par celui de l'espace fictionnel auquel s'identifient les personnages, en l'absence de toutes autres valeurs. Il est ensuite représenté chez Pinter par le langage banal, vidé de sa substance, sur lequel s'appuient les personnages pour masquer le vide de leur existence. A ce langage-coquille dans A Slight Ache correspond l'objet-coquille dans Lord of the Flies, symbole concret du vide, sur lequel les garçons rescapés de Golding fondent leur société. Enfin, l'écroulement sans surprise aucune des deux petites fragiles sociétés révèle l'homme obscur qui en est la cause directe et inaugure le règne du néant absolu. Celui-ci est figuré, dans Lord of the Flies, par l'obscurité qui couvre l'île à laquelle répond le comportement de plus en plus barbare des ex-écoliers anglais devenus méconnaissables. Dans $A$ Slight Ache, le royaume du néant prend une forme humaine à travers la personne du vieil aveugle sourd-muet qui fait effroyablement irruption dans le paisible appartement d'Edward et de sa femme. Par sa seule présence, Edward perd ce qui faisait son identité, son espace, révélant ainsi toute la précarité existentielle de l'être, dépourvu de substance identitaire réelle. Edward symbolise l'homme postmoderne sans ancrage identitaire et qui se perd dans les jeux des structures qui le guident et le font valser au gré de leurs caprices. Après donc la mort de Dieu «officiellement» annoncé par Nietzsche, une mort révélatrice du grand nihilisme, voici maintenant qu'advient la mort de l'homme, du sujet libre et transparent constatée par les poststructuralistes. C'est l'ère du nihilisme identitaire, autrement dit l'avènement de l'être arraché à tous ses fondements pour se noyer dans ce qu'Esslin appelle « l'océan du néant ». Lord of the Flies de Golding et $A$ Slight Ache de Pinter offrent des images vivantes et singulières de cette noyade du sujet moderne dans le néant de l'existence.

\section{References:}

\section{Corpus}

1. Golding W (1958). Lord of the Flies. London, Boston : Faber and Faber.

2. Pinter H (1991). Plays one : A Slight Ache et autres pièces.London, Boston : Faber and Faber.

\section{Autres travaux}

\section{Ouvrages}

3. Baudrillard J (2007). Pourquoi tout n'a-t-il pas déjà disparu ?Paris, Éditions de l'Herne. 
4. Begley V (2005). Harold Pinter and the Twilight of Modernism. Toronto : U of Toronto.

5. Beckett S (1958). Endgame. London : Faber \& Faber.

6. Camille F (2003). Dérives de la parole : les récits de William Golding. Paris : L'Harmattan.

7. Esslin M (1991). The Theatre of the Absurd. Hardmonsworth : Penguin Books.

8. Foster, S.W. (2002). Lord of the Flies. York Press. 322 Old Bro- mpton Road: London SW5 9JH.

9. FoucaultM (1966). Les Mots et les choses, Paris, Gallimard, collection « Bibliothèque des sciences humaines», 1966.

10........... (2001). Dits et écrits. 1954-1988, tome IV.Paris :Gallimard,

11. Gregory M (2009).Nature of Man in the First Six Fantasy Novels of William Golding.USA: XlibrisCorporation.

12. Howard S. Babb. (1970). The Novels of William Golding. Columbus: Ohio State University Press.

13. Kharoubi L (2007). Le visage de la scène : l'autre monde d'Harold Pinter et Emmanuel Lévinas, thèse de doctorat soutenue à l'université Paris IV Sorbonne le 7 décembre 2007.

14. Lacan J (1966). Les quatre concepts fondamentaux de la psychanalyse. Le Séminaire, Livre Xl (1964), texte établi par Jacques-Alain Miller, Paris, Éditions du Seuil.

15. Lercercle A (2006). Le Théâtre d'Harold Pinter. Stratégie de l'indicible : regard, parole, image. Paris : Klincksiek.

16. Nietzsche F (1969). Le livre du philosophe, Ill, trad. A.K. Marietti, Aubier Flammarion.

17. Niven, Alastair. (1980). Notes on Lord of the Flies. New York: Longman.

18. Pinter H (1983). Plays One. London : Methuen Drama.

19. Pruner M (2002). Les théâtres de l'absurde. Paris : Nathan.

20. Raby P (2001). The Cambridge Companion to Pinter (ed.). Cambridge : CUP.

21. Russ J (1995). La marche des idées contemporaines : un panorama de la modernité. Paris : Armand Colin.

22. Salem D (1993). Harold Pinter dramaturge de l'ambiguïté. Paris : Editions Denoël.

23. Tiger, Virginia M (1974). William Golding: The Dark Fields of Discovery. London: Calder \& Boyars.

\section{Articles}

24. Al-Saidi A H A (2012)."Savagery and the Heart of Darkness in William Golding's Lord of the Flies in Studies in Literature and 
Language Vol. 4, No. 1, Pp. 129-134.

25. Alnajm A L (2015). "The main theme in Lord of the Flies" in International Journal of English and Literature, Vol 6 (6), Pp. 98-102.

26. Cohn, R (1972). "The World of Harold Pinter" in Pinter: A Collection of Critical Essays. Ed. Arthur Ganz (New Jersey: Prentice-Hall, Inc., Pp. 78-92.

27. Devlin, K. J. (1994). "The eye and the gaze in Heart of Darkness: A symptomological reading" in MFS Modern Fiction Studies, 40(4), Pp. 711-735.

28. El Diwani F K M (2014). "So it goes : A Postmodern Reading of Kurt Vonnegut's Slaughterhouse-Five"in International Journal of English and Literature Vol 5 ( June), PP.82-90.

29. Fuegi Jo (1986). "The Uncertainty Principle and Pinter's Modern Drama" in Harold Pinter. Ed. Stephen Gale. Rutherford, New Jersey: Fairleigh Dickenson University Press, Pp. 202-207.

30. George J \& Raju R (November 2015). "Personal Accountability to Evil in William Golding's Lord of the Flies" in Mediterranean Journal of Social Sciences Vol 6 No 6 S2 MCSER Publishing, RomeItaly, Pp.174-178.

31. Guy Di Méo (2008). «Le rapport identité/espace. Eléments conceptuels et 'épistémologiques $»:\langle$ halshs-00281929>

32. Sathyaseelan S (2016). "Lord of the Flies an Allegory » in Journal of English Language and Literature, Vol 3 Issue 2, Pp. 99-103. 